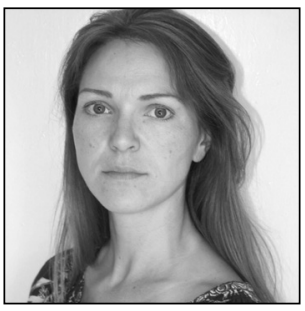

Mari-Liis Viirsalu

\title{
Die Verantwortungsstruktur bei der Privatisierung der Rehabilitationsleistungen im estnischen Sozialrecht
}

\section{Einleitung}

Die Kooperation zwischen dem Staat und den Privaten zur Erfüllung gemeinwohlorientierter Aufgaben ist in unterschiedlichen Ausprägungen in ganz Europa verbreitet. ${ }^{{ }_{1}}$ Das Gesundheitswesen und soziale Dienstleistungen stellen hier keine Ausnahme dar. ${ }^{{ }_{2}}$ Es wird von einem generellen Trend vom „Erfüllungsstaat“ in die Richtung „Gewährleistungsstaat“ gesprochen ${ }^{*}$, dessen näherer Inhalt rechtswissenschaftlich diskutiert wird. ${ }^{*} 4$

Mit diesen Entwicklungen sind unterschiedliche Erwartungen verbunden ${ }^{*}$, unter denen höhere Effektivität und erweiterte Selbstbestimmung der Bürger sowie die daraus folgende Befähigung zur Bewältigung ihrer Lebenslagen hervorgehoben werden können. Eine Gestaltung der sozialen Marktwirtschaft zur Erbringung von Sozialdienstleistungen wird auf der EU-Ebene ausdrücklich gefordert. ${ }^{*}$

1 H. Wollmann, G. Marcou, eds. The provision of public services in Europe: Between state, local government and market. Edward Elgar Publishing, 2010, S. 240; Zur Ausgestaltung der Kooperation zwischen dem Staat und den Privaten zur Erbringung öffentlicher und sozialer Dienstleistungen in historischer, länderübergreifender und rechtsvergleichender Sicht siehe: H. Wollmann, I. Koprić, G. Marcou, eds. Public and Social Services in Europe: From Public and Municipal to Private Sector Provision. Springer, 2016.

2 M. Huber, M. Maucher, B. Sak. Study on social and health services of general interest in the European union. Vienna/Brussels, European Centre for Social Welfare Policy and Research, ISS, CIRIEC, 2008, S. 227.

3 M. Huber, M. Maucher, B. Sak. Study on social and health services of general interest in the European union. Vienna/ Brussels, European Centre for Social Welfare Policy and Research, ISS, CIRIEC, 2008, S. 227; D. Ehlers, H. Pünder (Hrsg). Allgemeines Verwaltungsrecht: Mit Online-Zugang zur Jura-Kartei-Datenbank. Walter de Gruyter GmbH \& Co KG, 2016, $\S 10, \operatorname{Rn} 8$.

4 Knauff, der Gewährleistungsstaat: Reform der Daseinsvorsorge, 66-67. Voßkuhle VVDStRL 62, 2003, 266, 310ff.; C. Franzius. Gewährleistung im Recht: Grundlagen eines europäischen Regelungsmodells öffentlicher Dienstleistungen. Vol. 177. Mohr Siebeck 2009, S. $134 \mathrm{ff}$.

5 Übersichtlich: J. P. Schaefer, Die Umgestaltung des Verwaltungsrechts: Kontroversen reformorientierter Verwaltungsrechtswissenschaft. Vol. 256. Mohr Siebeck, 2016, 314-315; Burgi, Privatisierung öffentlicher Aufgaben-Gestaltungsmöglichkeiten, Grenzen, Regelungsbedarf, Gutachten D zum 67. Deutschen Juristentag. 2008, S 23. Erhältlich unter: http://www. bppp.de/media/file/65.DJT-Gutachten_Prof._Burgi.pdf

6 Entschließung des Europäischen Parlaments vom 05.07.2011 über die Zukunft der Sozialdienstleistungen von allgemeinem Interesse (2009/2222(INI)), P. 38. 
Aus wissenschaftlicher Sicht stellt sich mit der Einbindung gewerblicher Anbieter die Frage von der Verantwortungsteilung zwischen den Letztgenannten, dem Staat und den Bürgern. Besondere Aufmerksamkeit benötigt dabei die mögliche Gefährdung individueller Rechtsstellung des schutzbedürftigen Leistungsempfängers. ${ }^{*}$

Nach der Privatisierungsschwelle neunziger Jahre des 20. Jahrhunderts, geführt von „neo-liberalen“ Politik und „New Public Management"“* , gibt es in jüngerer Zeit Indizien für einen teilweisen „Rückschwung des Pendels ${ }^{\text {“*9}}$, der von einigen Wissenschaftlern als eine notwendige Balanzierung zwischen Vermarktlichung und Rechtswerte ausgewertet wird. ${ }^{*} 10$

In diesem Hintergrund ist es besonders interessant ein gegenläufiges Beispiel aus dem estnischen Sozialrecht vorzubringen. Dieser Beitrag widmet sich der kürzlich erfolgten Vermarktlichung von Leistungen der sozialen Rehabilitation der behinderten und arbeitsunfähigen Menschen (weiter bezeichnet als „Teilhabe“). ${ }^{* 11}$ Die Erneuerung trat im Verlauf des Kodifikationsvorhabens des estnischen Sozialrechts (und der parallel laufenden Arbeitsfähigkeitsreform) im Rahmen des ersten besonderen Teils des Sozialgesetzbuches - dem Fürsorgegesetz ${ }^{* 12}(\mathrm{FsG})$ - am 01.01.2016 in Kraft. Zu den wichtigsten Veränderungen auf der Strukturebene zählt die Auflösung des bisherigen Verwaltungsvertrages zur Erfüllung der öffentlichen Aufgabe, die in Übereinstimmung mit den Leitfaden der Europäischer Kommission ${ }^{* 13}$ durch eine alternative Lösung ersetzt wurde: alle Marktteilnehmer haben gleiche Möglichkeit eine Zulassung zu beantragen, um die Leistungen zu erbringen; der Leistungsberechtigte kann nunmehr zwischen den Anbietern frei wählen (s.g. system of choice); die Anforderungen an die Leistungserbringung, die Auflistung der einzelnen Leistungen sowie ihre Preise sind gesetzlich festgelegt. Auffällig ist, dass die vertraglichen Beziehungen zwischen dem Staat und den Leistungserbringern sowie zwischen den Leistungserbringern und den Leistungsberechtigten, anders als früher, privatrechtlich ausgestaltet wurden.

Ohne hier eine ganzheitliche Betrachtung aller mit der Reform verbundenen dogmatischen und verfassungsrechtlichen Fragen vorzunehmen, beschränkt man sich hier zum einen darauf, die umgeordneten Dreiecksverhältnisse anhand teleologischer und systematischer Argumente in die privatisierungstheoretischen Kontext zu setzen und dabei zu prüfen, inwieweit die Änderungen des Teilhaberechts den Voraussetzungen des Verwaltungskooperationsgesetzes entsprechen (2. Abschnitt). Später wird die infolge des Rollenwechsels des Staates umgeordnete Verantwortungsstruktur näher behandelt (3. Abschnitt) und bewertet, wie mit der heutigen Rechtslage der staatlichen Gewährleistungsverantwortung Rechnung getragen wird.

Da sich die estnische Jurisprudenz mit den Privatisierungsfragen der Sozialleistungen bisher nicht befasst hat, stütze ich mich auch auf den theoretischen Diskurs des deutschen Rechts. Eine Rechtfertigung dafür bietet - historisch bedingt ${ }^{*} 14$ - die ähnliche Ausprägung des estnischen und des deutschen Rechts hinsichtlich der klaren Trennung zwischen dem öffentlichen und privaten Recht und der damit verbundenen Fragen der Beteiligung Privater an der Erfüllung öffentlicher Aufgaben.

7 Näher zum Problemhorizont siehe z. B: P. Aerschot. Activation Policies and the Protection of Individual Rights: A Critical Assessment of the Situation in Denmark, Finland and Sweden, 2012.

8 Zur Definition siehe: F. Walther, New public management: The right way to modernize and improve public services?. International Journal of Business and Public Administration, vol 12, no 2, 2015.

9 T. Klenk, E. Pavolini, eds. Restructuring Welfare Governance: Marketization, Managerialism and Welfare State Professionalism, Edward Elgar Publishing, 2015, S. 259.

10 A. Benish, A. Maron, Infusing Public Law into Privatized Welfare: Lawyers, Economists, and the Competing Logics of Administrative Reform. Law and Society Rev, 50, 2016, S. 953-984. Die Entwicklung europäischer Wohlfahrtssyteme ist somit nicht einheitlich, sondern eher als „eigenartig“ und „pluralistisch“ zu gekennzeichnen. K. Schubert, P. de Villota, J. Kuhlmann, eds. Challenges to European Welfare Systems. Springer, 2016, S. 5.

11 Die Leistungen zielen auf Entwicklung der Fähigkeiten zur selbstbestimmtes Leben, zur Beteiligung in der Gesellschaft, zum Erlangen der Arbeitsfähigkeit im gewissen Umfang und auf die Entwicklung oder Wiederherstellung der Bereitschaft für die Aufnahme einer tragbaren Arbeit (Verweis 12).

12 Sotsiaalhoolekande seadus (Fürsorgegesetz) - RT I, 21.12.2016, 21 (auf Estnisch).

13 Arbeitsunterlage der Kommissionsdienststellen. Leitfaden zur Anwendung der Vorschriften der Europäischen Union über staatliche Beihilfen, öffentliche Aufträge und den Binnenmarkt auf Dienstleistungen von allgemeinem wirtschaftlichem Interesse und insbesondere auf Sozialdienstleistungen von allgemeinem Interesse. SWD(2013) 53 final/2. Brüssel, 29.4.2013. Erhältlich unter: http://ec.europa.eu/competition/state_aid/overview/new_guide_eu_rules_procurement_de.pdf

14 K. Merusk. Administrative Law Reform in Estonia: Legal Policy Choices and Their Implementation, Juridica International, 2004, No. 1, S. 61. 


\section{Von hoheitlicher Leistungserbringung zur materiellen Privatisierung?}

\subsection{Funktionale Privatisierung nach Maßgaben des Verwaltungskooperationsgesetzes?}

Bis Ende 2015 wurden die Rechtsverhältnisse zwischen dem Staat und den Leistungserbringern zur Erbringung der Teilhabeleistungen durch Verwaltungsverträge reguliert und fielen somit unter das Verwaltungskooperationsgesetz ${ }^{* 15}$ (VwKG), das die grundlegenden Voraussetzungen und das Verfahren für die Einbeziehung Privater in die selbstständige Erfüllung öffentlicher Aufgaben vorsieht. Die Privaten dürfen zur Erfüllung einer Verwaltungsaufgabe aufgrund Gesetzes, Verwaltungsaktes oder Verwaltungsvertrags beauftragt werden, wenn (1) die Erfüllung der Aufgabe von einer juristischen Person oder einer Einzelperson wirtschaftlich gerechtfertigt ist, unter Berücksichtigung der möglichen Kosten für die Beauftragung, mögliche Finanzierung und die Verwaltungsaufsicht; (2) die Beauftragung mit der Erfüllung einer Verwaltungsaufgabe die Erfüllungsqualität nicht verschlechtert; (3) die Beauftragung mit der Erfüllung einer Verwaltungsaufgabe die öffentlichen Interessen oder die Rechte der Betroffenen nicht schädigt (§ 5 Abs. 1 VwKG). Der Beauftragte wird Träger öffentlicher Gewalt. ${ }^{* 16}$ Die hoheitliche Aufgabenwahrnehmung des Leistungserbringers, der im Verhältnis zum Leistungsberechtigten als Behörde auftritt, entspricht weitgehend der deutschen Rechtsfigur der Beleihung. ${ }^{* 17}$

Es wird im Vorfeld der Privatisierung eine Analyse gefordert (Abs. 2) über die Massnahmen zur Gewährleistung der Kontinuität und Qualität der Erfüllung sowie über die rechtliche und faktische Wirkung für die Betroffenen. Die Entscheidung ist also mit einer erhöhten Begründungspflicht ${ }^{*}{ }^{* 18}$ verbunden. Die geordnete Analyse dient der Erfüllungsverantwortung die - zusätzlich zu der eigenständigen Verantwortung des Privatisierungssubjektes - auch nach der Beauftragung bei dem Staat verbleibt. Insoweit lässt sich auch von einer bei dem Hoheitsträger belassenen „Aufgabenzuständigkeit“ und „Aufgabenverantwortung“ zu sprechen. ${ }^{* 19}$

Die vorgestellten Anforderungen greifen nicht bei der Heranziehung von Privaten durch einen privatrechtlichen Vertrag, was sich damit rechtfertigen lässt, dass die Tätigkeit des privaten Akteurs in diesem Fall dem Verwaltungsorgan unmittelbar zugerechnet wird. ${ }^{* 2}$

Eine Prüfung, inwieweit die Änderungen des Teilhaberechts den Erfordernissen vom VwKG entsprechen, verursacht zunächst Verwirrung. Es werden zwar die personellen, sachlichen und informationellen Anforderungen gesetzlich festgelegt, da es aber an einer ausdrücklichen Zuweisung der Aufgabe an ein verpflichtetes Verantwortungssubjekt fehlt, kann nicht von einer gesetzlichen Beauftragung ausgegangen werden. Mit der Zulassung der gewerblichen Anbieter wird eine Möglichkeit, nicht gleich eine Betriebspflicht geschaffen.

Ein Abschluss des Verwaltungsvertrags wird vom Gesetzgeber ausdrücklich verweigert. ${ }^{{ }_{21}}$ Allerdings wird zwischen dem zur unmittelbaren Staatsverwaltung zugehörigen Sozialversicherungsamt und dem Leistungserbringer ein privatrechtlicher Vertrag geschlossen ${ }^{{ }^{22}}$, was die Frage aufwirft, ob der

15 Zur Entstehungsgeschichte vom Verwaltungskooperationsgesetz (RT I, 30.12.2015, 79, auf Estnisch) siehe: K. Merusk. Administrative Law Reform in Estonia, S. 61.

16 Begründung zum Gesetzesentwurf des Verwaltungskooperationsgesetzes (SE 474). (Auf Estnisch).

17 Siehe z. B. GVwR, 2006, § 12 Rn 106 (Schultze-Fielitz); Burgi, DJT S 40.

18 Vrg auch Stober, Privatisierung öffentlicher Aufgaben. Phantomdiskussion oder Gestaltungsoption in einer verantwortungsgeteilten, offenen Wirtschafts-, Sozial- und Sicherheitsverfassung? Neue Juristische Wochenschrift 2008/32, S 2308.

19 Schoch, Gewährleistungsverwaltung: Stärkung der Privatrechtsgesellschaft? NVwZ 2008, S. 246.

20 Laut § 3 Abs. 4 VwKG kann zur Erfüllung einer Verwaltungsaufgabe ein zivilrechtlicher Vertrag nur geschlossen werden, wenn das Gesetz nicht den Abschluss eines Verwaltungsvertrages vorsieht, wenn mit dem Vertrag die Rechte und Pflichten vom Nutzer der öffentlichen Dienstleistung oder von sonstigen Dritten nicht berührt werden, wenn der Staat oder die Gemeinden nicht von ihren Pflichten befreit werden und bei der Erfüllung der Aufgabe keine Hoheitsbefugnisse genutzt werden. Im Umkehrschluss ergeben sich aus der Vorschrift die Kriterien, wenn bei der Einbeziehung Privater ein Verwaltugsvertrag erforderlich ist.

21 Begeründungen zum Gesetzesenwurf (Seletuskiri sotsiaalhoolekande seaduse, tööturuteenuste ja -toetuste seaduse ning teiste seaduste muutmise seaduse eelnõu juurde), $693 \mathrm{SE}$, Anhang 1. Erhältlich unter: https://www.riigikogu.ee/ download/2ec35a2d-d1a1-4cfd-a38f-e2bcdaed3a28 (auf Estnisch).

22 So die Begründung zum Gesetzesentwurf 693 SE, S. 19. Erhältlich unter: https://www.riigikogu.ee/download/7a99e5ed62ba-420c-ae6c-7a985be44d92 (Auf Estnisch). 
Leistungserbringer aufgrund dieses Vertrages zur Bedarfsermittlung und -Planung sowie zur Leistungserbringung verpflichtet wird. Die Vorschrift überdeckt sich im Wesentlichen mit dem neueren Verständnis der deutschen Jurisprudenz zur theoretischen Konzept der selbstständigen Verwaltungshilfe. ${ }^{{ } 23}$ Ein Indiz für die Einordnung dieser Aufgabenübertragung als funktionale Privatisierung ist die fortbestehende staatliche Aufsicht über die Leistungserbringer. Andererseits, während für die Tätigkeit des Verwaltungshelfers ${ }^{*} 24$ die Behörde weiterhin verantwortlich bleibt, wird im vorliegenden Fall der Teilhabeprivatisierung die Erfüllungsverantwortung einschliesslich der Bedarfsermittlung und Hilfeplanung den Leistungserbringern überlassen. Die möglichen Streite über die Rechtsmässigkeit der Bedarfsermittlung, soweit von Leistungserbringern ausgeführt, sowie die Leistungsstörungen sind nunmehr im privatrechtlichen Rechtsverhältnis zu beseitigen - das verwaltungsrechtliche Widerspruchsverfahren wird ausgeschlossen. Eine Änderung ist im Gesetz nur daran zu erkennen, dass die privaten Leistungserbringer fortan gesetzlich zur Bereitstellung einer Ordnung für die Streitbelegung verpflichtet werden, ohne an dieses Verfahren genauere Anforderungen zu stellen.

Der Inhalt des Vertrags ist laut Gesetzes auf die Festlegung der Modalitäten der Kostenübernahme begrenzt ( $(65$ FsG), nicht auf die Übertragung der Aufgabenerledigung gerichtet. Ob mit diesem Vertrag eine Betriebspflicht bzw. Kontrahierungszwang im Verhältnis zur Klientel ${ }^{* 25}$ geschaffen wird, lässt sich aus dem Gesetz nicht entnehmen. Dadurch wird klar, dass die gewählte Kooperationsart durch § 3 Abs. 4 VwKG nicht umfasst wird.

\subsection{Materielle (Teil-)Privatisierung der öffentlichen Aufgabe?}

Die Umstrukturierung der Dreiecksbeziehungen ${ }^{* 26}$ im Teilhaberecht in Anlehnung an den bereits oben vorgestellten Leitfaden der Europäischen Kommission lassen sich daher anhand der Vorgaben des VwKG nicht erklären. Damit stellt sich die Frage, ob Teilhabe überhaupt noch als öffentliche Aufgabe angesehen wird und anzusehen ist. Beim näheren Anschauen zeigt sich tatsächlich, dass es sich aus Sicht des Gesetzgebers bei den Leistungserbringern nicht mehr um Erfüllern öffentlicher Aufgabe handelt. ${ }^{{ }^{27}}$ Diese Aussage bedarf aus mehrfacher Hinsicht kritischer Würdigung.

Hinsichtlich der Teilhabeleistungen besteht kein grundsätzliches Privatisierungsverbot. ${ }^{* 28}$ Der Staat verfügt über die „Kompetenzverteilungskompetenz“ zu entscheiden, ob er eine Aufgabe als öffentliche Aufgabe wahrnimmt, oder entsprechende hoheitliche Regulierung schafft, aufgrund deren die Privaten die Aufgabe wahrnehmen. ${ }^{* 29}$ Die Anerkennung einer Aufgabe als öffentliche Aufgabe setzt nicht zwingend eine Ausübung der Staatsgewalt aus. ${ }^{*} 30$

Unter den Aufgaben des zur unmittelbaren Staatsverwaltung gehörenden Sozialversicherungsamts ist auch die „Gewährleistung der Erbringung von Teilhabeleistungen“ genannt. Dem entspricht das subjektiv-öffentliche Recht des Bürgers auf Kostenübernahme für die privatrechtlich erbrachte Leistung (§ 56 FsG). Die ausdrückliche Verweigerung einer Erfüllung öffentlicher Aufgabe könnte bedeuten, dass der Gesetzgeber von einer Trennung von hoheitlicher Gewährleistungsverantwortung und einfacher Leistungserbringung ausgeht, so dass die Letztere von der öffentlichen Aufgabe nicht mehr umfasst wird. Diese Abspaltung hat zur Folge, dass der Staat sich von der Erfüllungsverantwortung zurückzieht und die Privaten bei der Leistungserbringung nicht eine formell öffentliche Aufgabe erfüllen, sondern die

\footnotetext{
Di Fabio, S. 590; Burgi DJT, S 34.

Näher zum Konzept der Verwaltungshilfe siehe: M. Burgi DJT, S. 41.

25 Zu unterschiedlichen Dimensionen der Nutzerstellung des Bürgers im Sozialrecht siehe: Rechtliche Dimensionen der Nutzerstellung im Sozialrecht, in: Gerhard Igl (Hrsg.), Verbraucherschutz im Sozialrecht. Sozialleistungsberechtigte als Verbraucher, Nutzer und Mitgestalter sozialer Leistungen: Auf dem Weg zu einem eigenständigen Verbraucherschutz im Sozialrecht, Berlin, 2011, S. 23.

26 Ausführliche Analyse der Dreicksbeziehunen vor und nach dem Reform siehe: M-L. Viirsalu., Eraõiguslik sotsiaalõigus: vastutuse muutumine sotsiaalse rehabilitatsiooni õigussuhete kolmnurgas, Juridica 2/2017 (Privatrechtliches Sozialrecht: Veränderung der Verantwortung in Dreiecksbeziehungen der sozialen Rehabilitation), S. 83-85.

27 Begeründungen zum Gesetzesenwurf (Seletuskiri sotsiaalhoolekande seaduse, tööturuteenuste ja -toetuste seaduse ning teiste seaduste muutmise seaduse eelnõu juurde), 693 SE, Anhang 1, S 4. Erhältlich unter: https://www.riigikogu.ee/ download/2ec35a2d-d1a1-4cfd-a38f-e2bcdaed3a28 (auf Estnisch).

28 Viirsalu, S. 89.

29 Parrest, Segadus mõistetes seoses avaliku võimu ülesannetega. - Juridica 2014/10, S. 737.

30 Urteil des Staatsgerichtshofes vom 14.12.2011, 3-3-1-72-11, P. 8.
} 
Aufgabe als ihre eigene wahrnehmen. ${ }^{*}{ }^{11}$ Dieses Konzept weicht von der bisherigen Auffassung des Staatsgerichtshofes ab. ${ }^{*}{ }^{32} \mathrm{Um}$ die Künstlichkeit der Trennung zu überwinden und dieses Konzept brauchbar zu machen, soll zu Instrumenten des Gewährleistungsstaates gewandt werden (siehe Abschnitt 3.1).

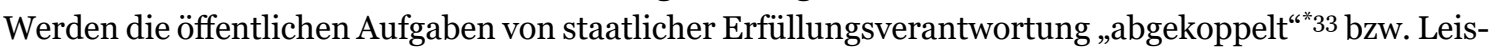
tungen aus dem Verwaltungsbereich herausgenommen ${ }^{*}{ }^{34}$, wandelt sich der Modus der Staatsaufgabenwahrnehmung, wobei die öffentliche Aufgabe bestehen bleibt. ${ }^{*} 35$

$\mathrm{Zu}$ diesem Punkt wird die Unterscheidung zwischen vorbereitender und ausführender Art der Einbeziehung Privater relevant. Die Leistungserbringer werden nicht nur bei der Ausführung der inhaltlichen Teilhabeleistungen tätig, sondern verfügen über einen beachtlichen Entscheidungsspielraum (der die Ausfüllung unbestimmter Rechtsbegriffe und einen hohen Prognoseanteil enthält) auch bei der Bedarfsfeststellung und Hilfeplanung (Umfang, Art, Länge und Häufigkeit der Leistungen). Die Einbeziehung Privater in die Entscheidungserfindung erfolgt in komplizierten Fällen, in denen nicht der hoheitliche Leistungsträger selbst, sondern der zum Leistungserbringer gehörige Rehabilitationsteam die Bedarfsermittlung durchführt und ihre Ergebnisse in einem Rehabilitationsplan zwingend festlegt. ${ }^{*} 6$ Formell erfolgt das Verfahren konsensuell im Rahmen des privatrechtlichen Leistungsvertrags, materiell ist wegen einer scharfen Wissensasymmetrie zwischen der behinderten Person und den Rehabilitationsexperten eine einseitig auferlegte Entscheidung anzunehmen. ${ }^{*} 37$

Gesetzlich ist die Ausführung der Bedarfsfeststellung die Aufgabe des Sozialversicherungsamts (§ 62 FsG). Eine explizite Ermächtigung der Leistungserbringer liegt nicht vor, obwohl eine gewisse Legitimation durch die Zulassung geboten wird. Die Aufgabenübergabe könnte als Verfahrensprivatisierung ${ }^{*} 38$ eingeordnet werden, die aber letztlich ausscheidet, weil nicht nur die Vorbereitung der Entscheidung an Private überlassen, sondern auch die eigentliche Entscheidungsverantwortung abgegeben wird, da die Bedarfsfeststellung und Hilfeplanung nicht durch eine behördliche Letztentscheidung bestätigt werden müssen. ${ }^{*}{ }^{39}$ Ein nachträgliches Informieren der Behörde gilt an dieser Stelle nicht als effektives Steuerungsinstrument.

Mit der Neuregelung ist eine deutliche Annäherung an das Gesundheitsrecht stattgefunden: auch der Behandlungsvertrag ist gemäss Schuldrechtsgesetzes ${ }^{*} 40$ ein privatrechtlicher Vertrag und über die nötige Behandlung wird eben auch im Rahmen dieses Vertrags privatrechtlich entschieden. Ein wesentlicher Unterschied besteht aber in den legitimierenden und die Rechtsstellung des Bürgers sichernden Schutzmassnahmen - die Beteiligung des Bürgers sichernden Schutzvorschriften sowie eine Möglichkeit zur Zweitbegutachtung und objektive vorgerichtliche Stelle für die Begutachtung der Leistungsqualität - die es für die soziale Rehabilitation derzeit nicht gibt.

Widersprüchlicherweise wird in der Gesetzesbegründung trotz der expliziten Aufgabenzuweisung das Fortbestehen der Gewährleistungsverantwortung hinsichtlich der Leistungsgewährung in Zweifel gezogen. In den Begründungen zum Gesetzesentwurf steht, dass der Markt selbst die angemessene Versorgung mit erforderlichen Dienstleistungen gewähre und der Staat anstatt der Naturalleistung lediglich zur Kostenübernahme verpflichtet bleibe. Das bietet auch eine Erklärung zur Nichtbeachtung der Anforderungen des $\S 5$ vom Verwaltungskooperationsgesetz.

31 In diese Richtung geht auch die in der Literatur neuerdings vertretene Auffassung, dass die Leistungserbringung nicht unbedingt eine öffentliche Aufgabe sein muss, wenn ihre Gewährleistung als öffentliche Aufgabe gilt. Parrest, S. 737. Teilweise wird diese künstliche Trennung einer Gesamtaufgabe auch in der Literatur abgelehnt: Knauff, S 81.

32 Der Staatsgerichtshof hat mehrmals die Auffassung vertreten, dass das Wesen der öffentlichen Aufgabe sich nicht durch eine private Durchführung ändert und das es um Erfüllung öffentlicher Aufgabe auch dann handelt, wenn eine Private vom zuständigen Organ ermächtigt oder verpflichtet wird, im öffentlichen Interesse eine solche Leistung zu erbringen, für die der Staat eine Letztverantwortung trägt. Siehe: Beschlsüsse des Staatsgerichtshofes vom 13.02.13, 3-3-1-49-12, P. 23 und vom 16.02.2010, 3-3-4-1-10, P. 5.

33 Schaefer, Die Umgestaltung des Verwaltungsrechts: Kontroversen reformorientierter Verwaltungsrechtswissenschaft. Vol. 256. Mohr Siebeck, 2016, S. 412.

34 Kämmerer, Privatisierung: Typologie-Determinanten-Rechtspraxis-Folgen. Vol. 73. Mohr Siebeck, 2001, S. 440.

35 Kämmerer, S. 439-440.

36 Näher Viirsalu, S. 91-93.

37 Zwar entfalten die gesetzlich gebotene Beteiligungsrechte gewisse legitimierende Wirkung, aber wesentlich ist die früher hoheitlich ausgeführte Bedarfsfeststellung unverändert geblieben.

38 Näher zum Konzept siehe: Burgi, DJT, S 33.

39 Viirsalu, S. 85.

40 Võlaõigusseadus (Schuldrechtsgesetz). RT I 2001, 81, 487 (auf Estnisch), Abschnitt 41 („Behandlungsvertrag“). 
Tatsächlich fehlt es an gesetzlich festgelegter Sicherstellung der Leistungserbringung wie es im deutschen Teilhaberecht zu finden ist. ${ }^{*}{ }^{4}$ Als einziges Zeichen der Letztverantwortung gilt die Regelung über die maximale Länge der Wartezeit (1 Jahr) ${ }^{*}{ }^{42}$, die entweder eine Anerkennung der Strukturverantwortung oder blindes Vertrauen des Gesetzgebers in Marktkräften bedeuten kann. Es fehlen jedenfalls Kriterien für ein flächendeckendes Vorhandensein der erforderlichen Leistungen sowie einer Betriebspflicht von gewerblichen Anbietern. Übernimmt der Staat die Garantenstellung nicht, wird es als eine rechtswidrige Aufgabenprivatisierung zu bewerten sein.

Materiell bewertet gibt es bei den Teilhabeleistungen ein enger Gemeinwohlbezug ${ }^{*} 43$ und damit das öffentliche Interesse ${ }^{*} 44$ sowie die verfassungsrechtliche Grundrechtsverbindung ${ }^{*} 45$, die nach Wesentlichkeitstheorie (ableitbar von § $3 \mathrm{GG}$ ) Argumente für die Anerkennung als öffentliche Aufgabe darstellen. Das estnische Grundgesetz beinhaltet im $\S 28$ soziale Grundrechte ${ }^{*} 46$, die durch die internationalen Verpflichtungen präzisiert und teilweise erweitert worden sind. ${ }^{*} 47$ Trotz breiten Entscheidungsspielraums des Staates zur Bewältigung der sozialen Risiken, darf die Bestimmung jedoch nicht in der Weise ausgelegt werden, dass der Staat zum bloßen Zahlstelle degradiert wird - es müssen auch notwendige Dienstleistungen vorhanden sein. ${ }^{*}{ }^{8}$ Anders als in Deutschland, wo die allgemeine Gewährleistungsverantwortung vornehmlich als theoretisches Konzept aus dem Sozialstaatsprinzip hergeleitet wird ${ }^{*} 49$, wird dieses Verständnis im estnischen Recht durch ein eigenartiges Paragraf des Grundgesetzes gestärkt: $\S 14 \mathrm{GG}^{*}{ }^{50}$ verpflichtet alle drei Gewalten zur Gewährleistung von Grundrechten und formt somit eine besondere Bindungswirkung ${ }^{*}{ }^{1}$, die zum Schutz der Grundrechte aktives Tätigwerden fordert.

\subsection{Bewertung}

Der Versuch, die umgeordneten Dreiecksverhältnisse anhand der theoretischen Privatisierungstypen ${ }^{*}{ }^{* 2} \mathrm{zu}$ systematisieren, scheitert einerseits an widersprüchlicher Gesetzgebung. Ohne dogmatische Fundierung erfolgte Änderungen bergen Elemente sowohl der funktionalen als auch der materiellen Privatisierung. ${ }^{*}{ }^{5}$ Andererseits wird die Erkenntnis bestätigt, dass die theoretischen Privatisierungsarten und ihre vielfältigen

41 Vrgl. § 23 SGB IX.

42 Im konkreten Fall sprechen auch systematische Argumente gegen eine Beschränkung auf die blosse monetäre Verpflichtung. Nämlich wird übersehen, dass das allgemeine Teil des Sozialgesetzbuches bereits die „Kostenübernahme“ als eine Sonderform der Naturalleistung definiert (SGB AT § 14). Der Anspruch auf Übernahme der Leistungskosten wäre auch nicht logisch zu realisieren, wenn es keine Dienstleistung gäbe. Zur analoge Auslegung der Kostenübernahme der Jugendhilfeleistungen im deutschen Sozialrecht siehe: Wiesner, SGB VIII, Kinder- und Jugendhilfe, 5. Auflage 2015, Vorbemerkungen, Rn. 16.

$43 \mathrm{Zu}$ den Zielen der Teilhabeleistungen siehe Verweis 11.

44 Zum öffentlichen Interesse aus Sichtweisen der Verwaltungslehre, Wirtschaftslehre, Philosophie und Rechtswissenschaft: G. Vonk. Social security as a public interest: A multidisciplinary inquiry into the foundations of the regulatory welfare state.12 Eur. J. Soc. 2010, S 2-15; Das öffentliche Interesse an Sozialdienstleistungen von allgemeinem Interesse wird auch auf der EU-Ebene bestätigt:„Neben seiner Aufgabe, die Finanzierung der Sozialdienstleistungen von allgemeinem Interesse sicherzustellen, ist der Staat insgesamt dafür verantwortlich, unter Wahrung der Zuständigkeiten der beteiligten Akteure das Funktionieren der Sozialdienstleistungen zu gewährleisten und ein hohes Qualitätsniveau aufrechtzuerhalten.“ - Stellungnahme des Europäischen Wirtschafts- und Sozialausschusses zu der „Mitteilung der Kommission: Umsetzung des Gemeinschaftsprogramms von Lissabon - Die Sozialdienstleistungen von allgemeinem Interesse in der Europäischen Union“, $\mathrm{KOM}(2006) 177$ endg.

45 G. S. Katrougalos. Constitutional limitations of Social Security Privatisation: A human rights approach. Eur. J. Soc. Sec. 12 (2010): 16.

46 § 28 Abs. 2 GG: „Jeder estnische Staatsbürger hat das Recht auf staatliche Unterstützung im Alter, bei Arbeitsunfähigkeit, Verlust des Ernährers oder in Notlagen. Die Art der Unterstützung, ihr Umfang und ihre Bedingungen sowie die Verfahren werden durch Gesetz bestimmt.“

47 Mit weiteren Hinweisen auf europäische Sozialversicherungsinstrumete siehe: E. Eichenhofer, Social security as a human right. Research handbook on European social security law. F. Pennings, G. Vonk (Eds), Edward Elgar 2015, S. 26 ff.

48 Viirsalu, S. 88. Vrg. Gegenmeinung am Beispiel des Sozialhilfedreiecks, nach der mit dem Übergang vom Sachleistungs- zum Geldleistungssystem die Gewährleistungspflicht entfälle: D. Meyer. Markt - Staat - Wettbewerb in der Sozialwirtschaft. Review of Economics 2008 (59) 2, lk 114-140.

49 Mit Ausnahme der bereichsspezifischen verfassungsrechtlichen Vorschriften zur Flugsicherung, Postdienste und Eisenbahnen (Art. GG Artikel 87d, GG Artikel 87e, GG Artikel 87f GG). Schoch, S. 243.

50 „Die Gewährleistung der Rechte und Freiheiten ist eine Pflicht der gesetzgebenden, vollziehenden und rechtsprechenden Gewalt wie auch der örtlichen Selbstverwaltungen.“

51 R. Alexy. Põhiõigused Eesti põhiseaduses. - Juridica 2001, Sonderausgabe, Abs. 2.1.2.

52 GVwR Bd I 2006, § 12 Rn 108ff (Schultze-Fielitz).

53 Genauer siehe: Viirsalu, S. 93. 
Unter- und Zwischenformen auch nicht völlig dafür taugen, die gezielten Änderungen zu erklären und zu bewerten. ${ }^{*} 54$ Am nächsten zur vorliegenden Konstellation, in der sich der Staat der Handlungsformen des Privatrechts bedient, wobei eine Garantenstellung hinsichtlich der Leistungserbringung behalten wird, steht die „unechte Aufgabenprivatisierung ${ }^{*} 55^{\circ}$. Ansonsten bietet die Privatisierungstheorie keine klaren Antworten, wie der Staat die ihm verbleibende Garantenstellung und Steuerung eines angemessenen Interessenausgleichs in Dreiecksbeziehungen ausführen muss.

Als Leitbild für die rechtliche Ausgestaltung der Teilhabe kann das Universaldienstmodell gesehen werden. Bei Universaldienstleistungen findet ebenso eine echte Verlagerung einer Leistung in den privaten Sektor statt. ${ }^{*}{ }^{6}$ Obwohl auch die Ausführung der Gewährleistung der Leistungserbringung nach wie vor von enger Kooperation zwischen dem Staat und den privaten Leistungserbringern geprägt ist, wird diese vom $\S 5$ VwKG, in dem von originärer Leistungspflicht des Staates ausgegangen wird, nicht erfasst. Allerdings gibt es keinen Grund, die Begründungspflicht im Vorfeld der Privatisierung im vorliegenden Fall auszuschließen. Vielmehr hätte es hinsichtlich des Grades des möglichen Steuerungs- und Kontrollverlustes ${ }^{*} 57$ noch strenger sein müssen. In Anbetracht der klaren Ausrichtung der EU auf die marktförmige Erbringung der Sozialleistungen sollten die aus $\S 5$ VwKG ausgehenden Anforderungen an die Begründetheit der Privatisierung (Wirtschaftlichkeit, Qualität, Nachhaltigkeit, Grundrechtsrelevanz für die Betroffenen sowie faktische und rechtliche Einwirkung auf ihre Rechte) auf Fälle, wie diese hier ausgedehnt werden. Als allgemeiner Grundsatz soll sie nicht nur die Exekutive, sondern auch den Gesetzgeber selbst binden.

Der Übergang zur Wettbewerbsstruktur und damit bewirkte Loslösung von dem VwKG hat zur Folge, dass der Leistungserbringer nicht mehr als Träger öffentlicher Gewalt auftritt und damit keine unmittelbare Verbindung zu Grundrechten und rechtsstaatlichen Verfahrensgarantien begründet wird. Das gilt nicht für Bedarfsfeststellung, deren Durchführung nach wie vor als Verwaltungsaufgabe geregelt wird und zur Übertragung an Private dem VwKG unterliegen muss.

\section{Wettbewerbliche Leistungserbringung durch Regulierung und Gewährleistungsverantwortung}

\subsection{Inhalt der Gewährleistungsverantwortung}

Der Rollenwechsel von providing zu enabling, d. h. das wettbewerbliche Tätigwerden der Privaten zur gemeinwohlförderlichen Wahrnehmung ${ }^{*}{ }^{8}$ der Leistungspflichen und die Befreiung des Staates von der Erfüllungsverantwortung, lassen sich klarer mit den Begrifflichkeiten der Regulierung und Gewährleistungsverantwortung beschreiben, weil sie die konkreten Handlungsformen und Steuerungsinstrumente bereitstellen.

„Regulierung “* ${ }^{\text {“*9 }}$ wurde ursprünglich durch den Zweck der (bloßen) Schaffung der Bedingungen zum Wettbewerb bzw. Ermächtigung der Marktkräfte beschrieben. In neuerer Literatur zum Regulierungsrecht wird allerdings auch der Zweck der Gemeinwohlsicherung in den Regulierungsbegriff einbezogen. ${ }^{* 60}$ So wird der marktwirtschaftlicher Wettbewerb als Instrument der Gemeinwohlverwirklichung eingesetzt. ${ }^{* 61}$ So verstanden sind die Regulierung und Privatisierung zwei Seiten einer Medaille: zur Erfüllung der nach der Privatisierung erhalten gebliebenen Gewährleistungspflicht wird primär Regulierungsinstrumentarium eingesetzt (s.g. Privatisierungsfolgerecht ${ }^{*}{ }^{* 2}$ ). Die Ausführung der Gewährleistungsverantwortung setzt eine klare Verantwortungsteilung aus. ${ }^{*}{ }^{6}$

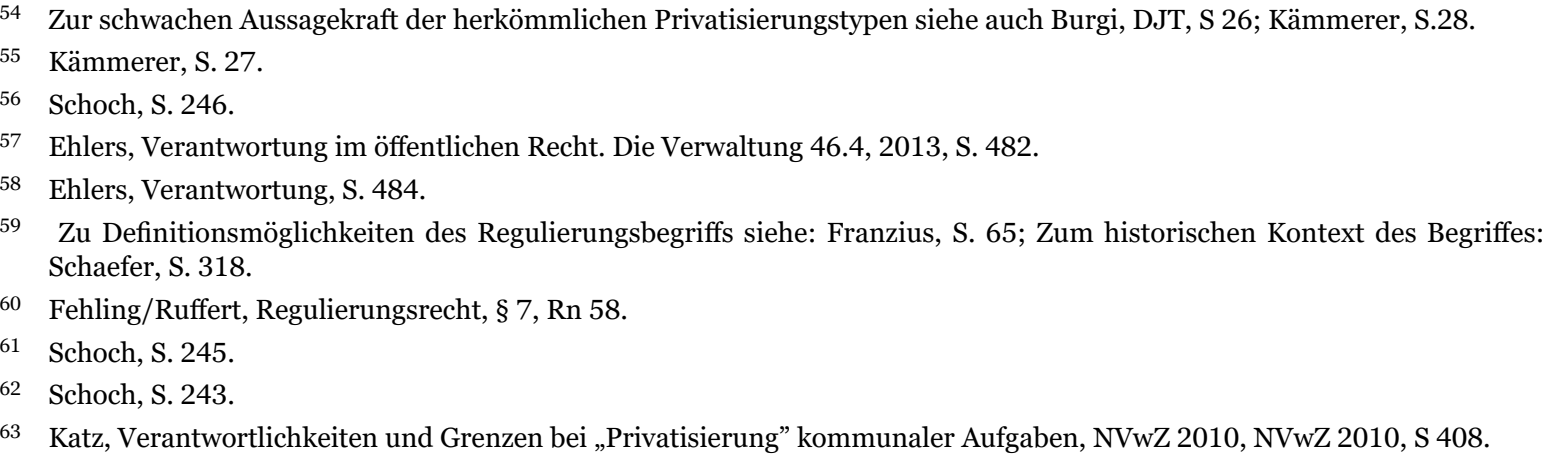


Obwohl mit der Privatisierung oft die Entlastung des Staates bezweckt wird, stellt die Gewährleistungsverantwortung dem Staat umfangreiche Anforderungen an Steuerung, Aufsicht und Finanzierung, die nicht unbedingt zur Entlastung führen. Jede Abstufung der Verpflichtungen bedarf einer Rechtfertigung aus dem Grundgesetz.

Daher liegt es nahe, dass die Verpflichtung zum Schutz der Rechte betroffenen Personen und die Erreichung der im öffentlichen Interesse liegenden Ziele als die wichtigsten inhaltlichen Elemente der Gewährleistungsverantwortung anzusehen sind. ${ }^{*} 4$ Dementsprechend trägt der Staat anstatt einer Erfüllungspflicht nun eine Ergebnisverantwortung. ${ }^{*} 65$

Als verfahrensrechtliche Elemente der Verantwortung gilt die Gewährleistung von Objektivität und Neutralität ${ }^{* 66}$, was die Gestaltung einer eigenständigen Verfahrensordnung voraussetzt, weil das Verwaltungsverfahrensrecht nicht unmittelbar anzuwenden ist. Die Objektivität und Neutralität bei der Bedarfsfeststellung und Hilfeplanung wird schon durch § 28 GG gefordert, zumal die Art und der Umfang der sozialen Hilfen dem Gesetzesvorbehalt unterliegen.

Um die Erreichung der Ziele sicherstellen zu können, bedarf es hinreichender staatlicher ${ }^{*} 67$ oder spezieller, auf die Gemeinwohlsicherung und auf den Schutz der Rechte Betroffener gerichteter Gewährleistungsaufsicht. ${ }^{* 68}$ Bei der sozialen Teilhabe ist die Staatsaufsicht erhalten geblieben, obwohl im Gesetzesentwurf lediglich eine auf Gefahrenabwehr und -Vorsorge gerichtete Überwachung der Wirtschaftstätigkeit vorgeschlagen wurde. Im Jahr 2018 wird in Estland das freiwillige europäische Qualitätsrahmen für Sozialdienstleistungen ${ }^{* 69}$ für die Leistungserbringer verbindlich gemacht. Inwieweit dieses, dogmatisch zu Eigenüberwachung gehörige Instrument ${ }^{*} 70$ das Fortbestehen der staatlichen Aufsicht beeinflusst oder ihre Lücken effektiv kompensieren kann, ist abzuwarten.

Da die Privatisierung der Leistungserbringung meist mit den Vorteilen des Wettbewerbs gerechtfertigt wird, soll auch die Iniziierung und Moderierung des Wettbewerbs ${ }^{*}{ }^{* 1}$ zur Gewährleistungsverantwortung gezählt werden.

Letztlich - und hier ist der Gesetzgeber in der Gesetzesbegründung falsch ausgegangen - trifft den Staat im Falle des Marktausfalles eine Auffangsverantwortung ${ }^{*}$, bis der Markt wieder handlungsfähig wird. Dies ist allerdings mit erheblichen praktischen Schwierigkeiten verbunden, falls der Staat von der Erfüllung der Aufgabe lange zur Seite geblieben ist. ${ }^{*} 73$ Es ist wohl eine Frage mit Praxisrelevanz, weil die Eigenart der Sozialleistungen diese (besonders im ländlichen Raum) hinsichtlich der Marktausfälle verletzlicher macht als in sonstigen Wirtschaftsbereichen. ${ }^{*} 74$

\subsection{Bewertung}

Die grundlegenden Änderungen in der Verantwortungsstruktur infolge der Privatisierung wirken in mehrfacher Hinsicht zu Lasten der besonders schutzbedürftigen Leistungsempfänger aus. Unzulässigerweise ist es zur Rückführung der öffentlich-rechtlichen Regelungsdichte gekommen, ohne dass mit dem Wechsel des Rechtsregimes zugunsten des Privatrechts nötige Schutzvorschriften geschaffen worden

64 Burgi, DJT, S. 101-102.

65 Beteiligung Privater an der Wahrnehmung öffentlicher Aufgaben und staatliche Verantwortung, S. 311 (A. Vosskuhle); Schoch, S. 245.

66 Burgi, DJT, S. 103.

67 Schaefer, S. 412.

68 B. Schmidt am Busch. Gewährleistungsaufsicht zur Sicherstellung privater Aufgabenerledigung. Eine dritte Kategorie zwischen Staatsaufsicht und Wirtschaftsaufsicht. - Die Verwaltung 2016 (49) 2, S. 205-232. Aber auch schon früher: Vosskuhle, Beteiligung Privater, S. 321.

69 Ausschuss für Sozialschutz 2010, SPC/2010/10/8 final, erhältlich unter: http://ec.europa.eu/social/BlobServlet?docId=6 140\&langId $=$ en

70 Grundlagen des Verwaltungsrechts. Band III: Personal, Finanzen, Kontrolle, Sanktionen, Staatliche Einstandspflichten. H. Hoffmann-Riem, E. Schmidt-Aßmann, A. Voßkuhle (Hrsg.). Verlag C. H. Beck 2013, § 45, Rn 199 (B. Huber).

71 Burgi, DJT, S. 105; Schaefer, S 412, 322.

72 Beteiligung Privater an der Wahrnehmung öffentlicher Aufgaben und staatliche Verantwortung, S 326 (A. Vosskuhle); Schaefer, S. 412; Franzius, S. 125; Ehlers, S. 484.

73 Gleiche Befürhtungen äußern: Franzius, S. 634; H. Trute. - G. Schuppert. Jenseits von Privatisierung, S. 34.

74 So R. M. Blank, R (2000). When can public policy makers rely on private markets? The effective provision of social services. The Economic Journal, 110(462), 49. 
wären. Auch ist es merkwürdig, dass diese Änderungen nicht schon früher Aufmerksamkeit der Juristen gewonnen haben.

Die Lücken können allerdings durch weise Regulierung und Wahrnehmung der Gewährleistungsverantwortung geschlossen werden. Eine mangelnde Grundrechtsverbindung lässt sich durch gesetzliche oder vertragliche Verpflichtungen begründen. ${ }^{*}{ }^{75}$ Auch beim s.g. system of choice kann und muss eine staatliche Letztverantwortung bestimmt sein sowie gesetzlich sichergestellt werden, dass ausreichende Leistungen zu vertretbaren Preisen auf dem Markt vorhanden und zugänglich sind. Ähnlich zu den anderen nach dem Universaldienstmodell ausgestalteten Leistungen muss eine Betriebspflicht bzw. Kontrahierungszwang bestehen.

Bei der Bedarfsfeststellung kommt in besonderer Weise die Anforderung zum objektiven und neutralen Verfahren zum Ausdruck, da die Entscheidung eine unmittelbare Wirkung auf die grundrechtliche Positionen der Teilhabeberechtigten hat und - um das öffentliche Interesse zu betonen - öffentliche Mittel eingesetzt werden. Es ist deshalb eine genauere „rechtsstaatliche Vorkonturierung“ im Sinne einer Verfahrensgestaltung zum Schutze des Leistungsberechtigten nötig. Zusätzlich muss eine Möglichkeit der Zweitbegutachtung und eine neutrale vorgerichtliche Streitstelle geschaffen werden. ${ }^{*}{ }^{76}$ Hinsichtlich der Rechtschutzmöglichkeiten ist bedauerlich, dass auf die Privatrechtsregime umgeschaltet worden ist, ohne die schutzbedürftigen Leistungsempfänger oder ihre Vertretungsorganisationen dafür vorzubereiten. Zusätzlich wäre an eine Zulässigkeit der Verbandklage zu denken.

Die oben betonte klare arbeitsteilige Verantwortungsteilung muss zukünftig verhindern, dass die vom Sozialversicherungsamt hoheitlich ausgeführte Bedarfsfeststellungen (in „einfachen Fällen“) und bei den Leistungserbringern erfolgte privatrechtliche Bedarfsfeststellung (in „komplizierten Fällen“) unterschiedlicher Gerichtsbarkeit und Verfahrensordnungen unterfallen, was die Bürger mit voraussichtlich höheren Schutzbedürftigkeit wegen Privatrechtsregime schlechter stellt.

Um weiterhin von der Wissenspotential des Privatsektors zu profitieren, wäre es grundsätzlich denkbar, die Bedarfsfeststellungsaufgabe im größeren Umfang den Privaten zu überlassen, vorausgesetzt, dass damit die demokratische Verantwortlichkeit gesichert werden kann. Kritisch betrachtet muss man zugleich hinnehmen, dass ein gewisser Verlust an staatlicher Steuerungsmöglichkeit doch unvermeidbar ist. ${ }^{*} 77$ Deswegen ist der Gesetzgeber aufzurufen, im Vorfeld jeder Privatisierungsentscheidung sorgsam der erhöhten Begründungspflicht nachzukommen und nüchtern zu bewerten, ob die Eigenart der Leistung (deren ökonomische Attraktivität) und die Schutzbedürftigkeit der Betroffenen eine Überlassung der Leistung auf den Markt rechtfertigen. ${ }^{*} 8$ Eine Abwägungshilfe bietet dafür auch die aus dem Rechtstaatsprinzip abgeleitete Wesentlichkeitstheorie, die je nach Intensität des Grundrechtsbezugs eine engere Einbeziehung öffentlicher Verwaltung fordert.

\section{Zusammenfassung}

Mit der Umstrukturierung von Dreiecksbeziehungen der sozialen Rehabilitation wurde ein Marktmodell ohne die staatliche Erfüllungsverantwortung geschaffen. Da die Leistungserbringung durch gewerbliche Anbieter nicht mehr als Erfüllung öffentlicher Aufgaben gewertet wird, greifen auch die durchaus nötigen Anforderungen des Verwaltungskooperationsgesetzes an Kontinuität, Qualität und Wirtschaftlichkeit der Leistungen sowie die Beachtung der Rechte der Betroffenen und des öffentlichen Interesses nicht mehr, obwohl es materiell weiterhin um gemeinwohl- und grundrechtsrelevante Aufgaben geht. Der Gesetzgeber wird aufgerufen, der nach dem Rollenwechsel dem Staat verbliebenen Gewährleistungsverantwortung Rechnung zu tragen und legislative Lücken zu schließen. Die Problematik der individuellen Rechtsstellung des Leistungsberechtigten, der heute im asymmetrischen Leistungserbringungsverhältnis als Verbraucher zwischen dem öffentlichen Recht und dem Privatrecht steht, bedarf einer tiefgehenden Behandlung in weiteren Beiträgen.

75 Vosskuhle, Beteiligung Privater, S. 319. Jedoch stehen die Grundrechte der gewerblichen Leistungserbringer den Rechten des Bürgers gegenüber, was ihre praktische Durchsetzung erschweren kann.

76 Nichtwirtschaftliche Dienstleistungen vom allgemeinen Interesse sind vom Zuständigkeitsbereich der vorgerichtlichen Streitbelegungsinstanz beim Verbraucherschutzamt ausgegrenzt. Die Analyse, ob die Tätigkeit von Rehabilitationserbringern unter diesen Begriff fällt, kann an diesem Ort nicht geleistet werden.

77 So im Ergebnis auch: Schoch, S. 245, 247.

78 Kriterien für solche Prüfung bietet: R. M. Blank. When can public policy makers rely on private markets? The effective provision of social services. The Economic Journal, 110, 2000, S. 462. 